\title{
Application of fast discrete wavelet transformation on the basis of spline wavelet for loosening correlation of sequence of data in mass service theory
}

\author{
Blatov I.A., Gerasimova U.A., Kartashevskiy I.V. \\ Povolzhskiy State University of Telecommunications and Informatics, Samara
}

\begin{abstract}
The task of loosening of correlation of sequence of strongly correlated random variables within the mass service theory is set. The algorithm of application of spline wavelet for loosening of correlation of sequence of strongly correlated random variables is described. Properties of the matrixes received as a result of application of transformation algorithm are studied. Results of numerical experiment studies are given.
\end{abstract}

Keywords: spline wavelets, decorrelation, fast discrete wavelet transformation

Citation: Blatov I.A., Gerasimova U.A.,Kartashevskiy I.V. Application of fast discrete wavelet transformation on the basis of spline wavelet for loosening correlation of sequence of data in mass service theory. Proceedings of Information Technology and Nanotechnology (ITNT-2015), CEUR Workshop Proceedings, 2015; 1490: 242-245. DOI: 10.18287/1613-0073-2015-1490-242245

\section{Introduction}

Strong correlation of sequence of random variables can create considerable problems in the solution of mass service theory. Let $X=\left(x_{0}, \ldots, x_{n}\right)^{T}$ be some vector with known correlation matrix $A$. It is required to analyze the traffic characterized by vector $X$ taking into account it correlation properties.

The traffic as random process is known to have self-similar properties, which indirect sign is the existence of heavy residuals, i.e. big redundancy of the appropriate integral functions of distribution. Therefore in such situation the method of preliminary execution of some orthogonal transformation determined by matrix $T=\left(t_{i j}\right)$, which purpose is elimination or lowering of correlation of basic data is often used. The application of up-to-date analysis from the mass service theory concerning vector $\tilde{X}=T X$, but not vector $X$, will proves to be more effective.

It is possible to eliminate correlation and to receive the best result by means of application of Karhunen-Loeva transformation. However creation of such basis is a very resource-capacious task. In this case matrix $T$ consist of eigenvectors of matrix $A$. The resultant correlation matrix will be of a diagonal type. However this method has some drawbacks such as: absence of fast algorithms of computation; dependence 
on the structure of matrix $A$. Therefore the task pf creation of more available bases in which the correlation can be, if not eliminated, but weakened essentially, is actual. In this report the spline wavelet is used.

\subsection{Elaboration of the system of semiorthogonal spline wavelets}

Let $[a, b]$ be a random interval, $m \geq 1$ be integer, $n_{0}$ be such an integer that $2^{n_{0}}<2 m+1<2^{n_{0}+1}$ and $k$ be such an integer that $2^{k}>2 m-1$. Let us consider the family $\Delta=\left\{\Delta_{n}, n=n_{0}, n_{0}+1, \ldots\right\}$ of partitions of the interval $[a, b]$ with the constant step $h=h_{n}=(b-a) / 2^{n}$. Let us define $S\left(\Delta_{n}, m, k\right)$ as the combination of spline wavelets, where $m$ is a power and $k$ is a degree. On each partition, we consider a space of splines $L_{n}=S\left(\Delta_{n}, m-1,1\right)$. Then, for each $k \geq n_{0}$, space $S\left(\Delta_{n}, m-1,1\right)$ can be represented as direct sum $L_{k}=L_{n_{0}} \oplus W_{n_{0}+1} \oplus W_{n_{0}+2} \oplus \ldots \oplus W_{k}$, where $W_{k}$ denotes the orthogonal complement of $L_{k-1}$ up to $L_{k}$ space. The desired wavelet basis is the result of combination of the basis in $L_{n_{0}}$ and all the bases in spaces $W_{n}, n_{0} \leq$ $n \leq k$.

Let $i \geq 0$ be a such a fixed integer that the interval $\left[x_{i}^{n-1}, x_{i+2 m-1}^{n-1}\right]$ lies within $[a, b]$. Function is computes according to formula

$\psi_{i, n}(x)=\sum_{j=2 i}^{2 i+3 m-2} \alpha_{j} \phi_{j, n-1}$

where $\phi_{j, n-1}$ normalized B-spline. The $\alpha_{j}$-coefficients are defined according to $\left(\psi_{i, n}(x), \phi_{k, n-1}\right)=0, k=i-m+1, i-m+2, \ldots, i+2 m-2$.

The combination of elaborated wavelet functions is resulted by shifting of the only function according to formula $\psi_{i, n}(x)=\psi_{0, n}\left(2^{n-n_{0}} x-i(b-a) / 2^{n_{0}-1}\right)$.

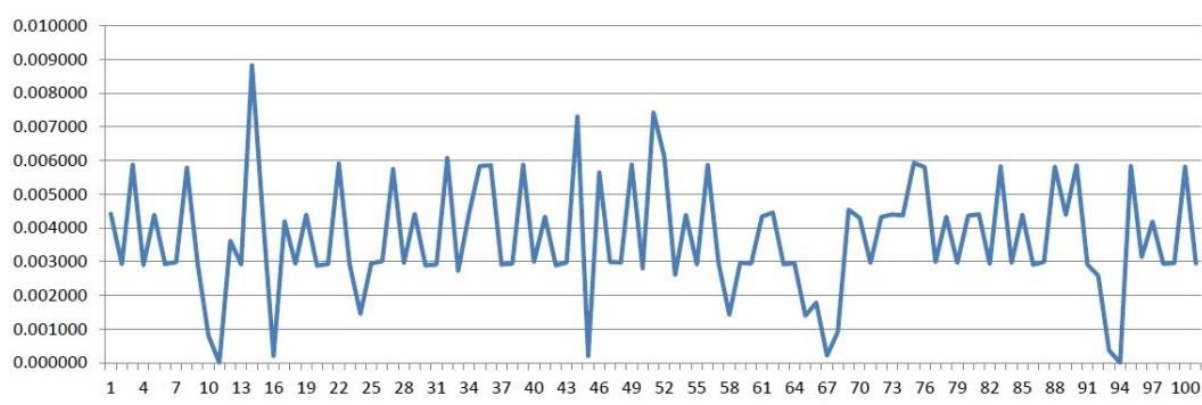

Fig. 1. - Sequence of experiment's data $X$

1.2. Fast discrete wavelet transformation in the space of spline wavelets on the finite interval

The direct transformation consist in the search of wavelet coefficients $d_{0 j}$ and $c_{i j}$.

$\left\{d_{0 j},-m+1 \leq j \leq 2^{n_{0}-1}\right\} \cup \cup_{i=1}^{k-n_{0}}\left\{c_{i j},-m+1 \leq j \leq 2^{n_{0}+i-1}-m\right\}$

According to known function $f=\left\{f_{i j}\right\}, 0 \leq i \leq 2^{k}-1,1 \leq j \leq s$. 
The inverse transformation consist in reconstruction of all values of function $f_{i j}, 0 \leq i \leq 2^{k}-1,1 \leq j \leq \mathrm{s}$ by $\left\{f_{i j}\right\} \in \tilde{S}\left(\Delta_{k}, m-1,1\right)$ according to the known set of wavelet coefficients

$$
f=\sum_{j=-m+1}^{2^{n_{0}-1}} d_{0 j} \phi_{j, n_{0}}+\sum_{i=1}^{k-n_{0}} \sum_{j=-m+1}^{2^{n_{0}+i-1}-m} c_{i j} \psi_{j, n_{0}+1}
$$

For more details on the a.m. items please consult [1].

\section{Numerical experiment}

We made an experiment on loosening of correlation of data represented by sequence $Y$ consisting of 9999 random values, each represents the time of traffic processing in the system ${ }^{1}$.

To the initial experiments data $X$ we applied direct fast discrete transformation on the basis of the linear spline.

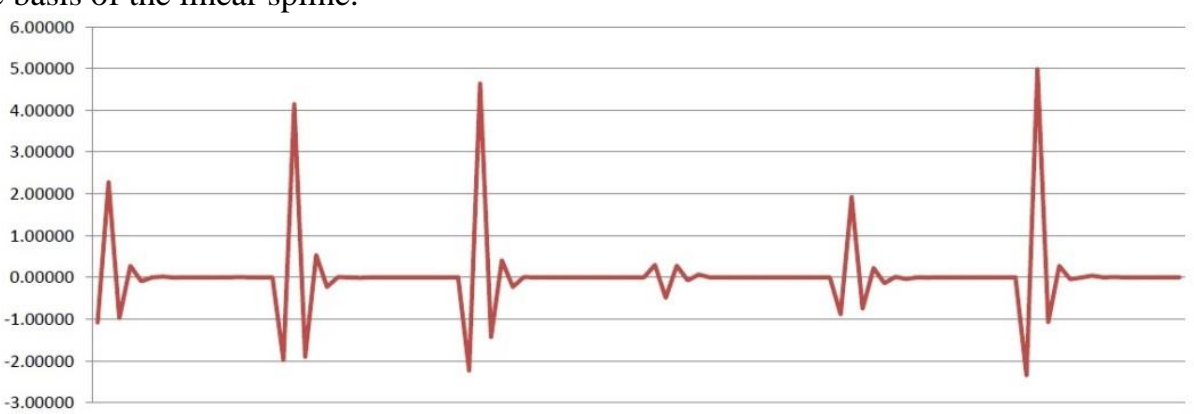

Fig. 2. - Sequence of data $\tilde{X}$ (after direct transformation)

The correlation coefficients were calculated on the basis of the experimental data $X$ (for more details see [3]):

$R_{k}=\frac{V_{k}}{D}$

where

$V_{k}=\frac{1}{n-k} \sum_{i=1}^{n-k}\left(Y_{k}-X\right)\left(Y_{i+k}-X\right)$

$D=\frac{1}{n} \sum_{i=1}^{n}\left(Y_{i}-X\right)^{2}$

$X=\frac{1}{n} \sum_{i=1}^{n} Y_{i}$

1 Problem definition about decorrelations and data for experiment were provided I.V. Kartashevsky 


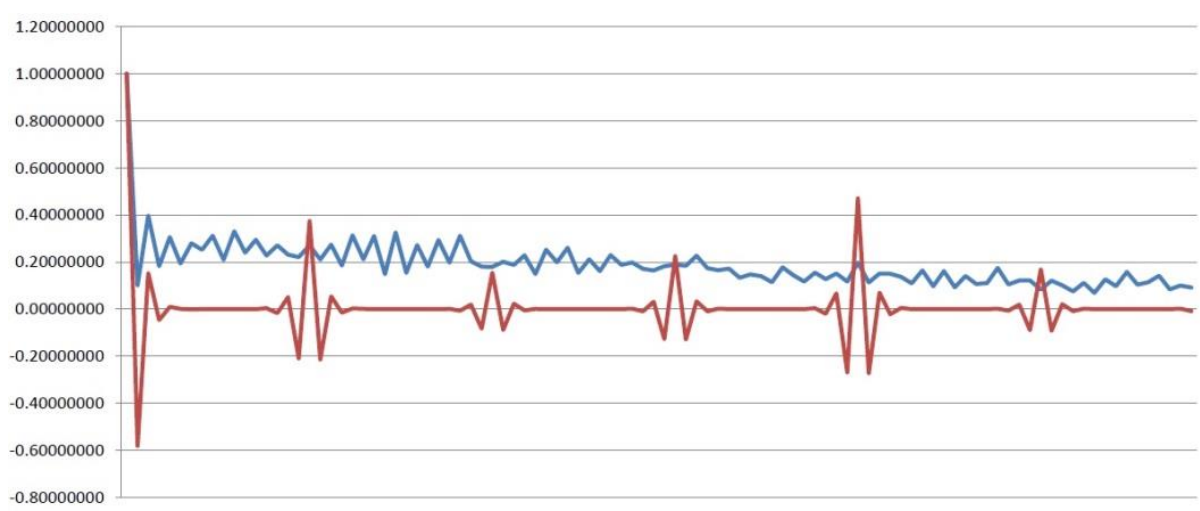

Fig. 3. - Correlation coefficients for experiment data and for transformed data

The sums of modules of correlation coefficients are calculated:

$R_{k}: \sum_{i=0}^{k-1}\left|r_{i}\right|=18.963$

$\tilde{R}_{k}: \sum_{i=0}^{k-1}\left|\widetilde{r}_{l}\right|=5.344$

The correlation is seen to have reduced by more than by 3.5 times. The similar result was received for square and cubic splines.

\section{Summary}

In the conclusion we would like to stress that the application of fast discrete algorithm of wavelet transformation in the space of spline wavelet allows loosening the correlation of sequence of strongly correlated random variables. That is confirmed by the data obtained in the numerical experiments.

\section{References}

1. Blatov IA, Rogova NV. Fast wavelet-transform in the space of discrete polynomial semiorthogonal splines. Computational Mathematical and Mathematical Physics, 2013; 53(5): 727-736.

2. Kartashevsky IV. Application Lindley equation for correlation traffic processing. Journal Electrosvyaz, 2014; 12: 41-42.

3. Kartashevsky IV. Calculation of correlation coefficients of times intervals in sequence of events. Journal Electrosvyaz, 2012; 10: 37-39.

4. Umnyashkin SV, Kochetkov ME. Analysis of efficiency of using orthogonal transform for digital coding of correlation data. Electronic, 1998; 6:79-84.

5. Myasnikov VV. Effective algorithm of calculation of local discrete wavelettransformation. Computer Optics, 2007; 31(4): 86-94. [in Russian] 